\title{
Laparoscopic Placement of Hepatic Artery Infusion Pumps: Technical Considerations and Early Results
}

\author{
Jun Cheng, MD, PhD, Dennis Hong, MD, Guojing Zhu, MD, Lee L. Swanstrom, MD, and \\ Paul D. Hansen, MD
}

\begin{abstract}
Background: Laparoscopic hepatic artery infusion pump (LHAIP) placement is a novel treatment option for patients with colorectal liver metastases. This study investigates technical difficulties with regard to variant hepatic arteries and the preliminary outcomes for patients treated with LHAIP placement.

Methods: Between March 1998 and January 2003, 38 patients with colorectal metastases confined to the liver, 35 (92\%) of who had prior systemic chemotherapy that failed, were treated with LHAIP.

Results: Twelve patients (32\%) had LHAIP placement only, and $26(68 \%)$ had pump placement combined with laparoscopic radiofrequency ablation (LRFA; 24 patients) and/or liver resection (2 patients). Variant hepatic arterial (HA) anatomy was present in 18 patients (47\%). The presence of a variant HA did not increase pump complications, operative time, or blood loss $(P \geq .20)$ or decrease the functional time of pump use $(P=.91)$ in comparison with normal anatomy. In all patients with a variant HA, laparoscopic ligation of the variant vessel and/or cannulation of nongastroduodenal artery resulted in complete hepatic perfusion. Three misperfusions identified intraoperatively with use of methylene blue injection were corrected by laparoscopic ligation (two) or postoperative angioembolization (one). Postoperative pump radionuclide flow studies confirmed isolated hepatic artery infusion in all cases. There was a $13 \%$ pump-related complication rate. During a median follow-up of 11 months ( 0.5 to 35.5 months), the actuarial rate of overall survival was $47 \%$ and the estimated median survival time was 17.5 months.
\end{abstract}

Conclusions: LHAIP placement is technically feasible, and variant HA is not associated with increased pump complications or decreased pump functional time.

Key Words: Colorectal liver metastases-Hepatic artery infusion pump—Laparoscopy.

Each year in the United States, approximately 150,000 people are diagnosed with primary colorectal cancer. About 80,000 of these patients will develop liver metastases, and half will be isolated to the liver. Although surgical resection is widely accepted as the "gold standard" treatment for isolated liver metastases, fewer than $25 \%$ of patients are candidates for the procedure. ${ }^{1}$ This

Received May 13, 2003; accepted February 2, 2004.

From the Department of Minimally Invasive Surgery, Legacy Health System, Portland, Oregon. Presented at the 4th America Congress of American Hepato-Pancreato-Biliary Association, Miami Beach, Florida, February 27 to March 2, 2003.

Address correspondence and reprint requests to: Paul D. Hansen, MD, General Surgery, TC-29260, University of Michigan Health System, 1500 E. Medical Center Drive, Ann Arbor, MI 48109; e-mail: pdhmd@aol.com.

Published by Lippincott Williams \& Wilkins @ 2004 The Society of Surgical Oncology, Inc. leaves up to 30,000 patients per year with colorectal liver metastases who will have systemic chemotherapy as their only treatment alternative.

Implantable pumps were first introduced in the 1980s. ${ }^{2}$ The rationale for delivering chemotherapy through the hepatic artery (HA) is that hepatic metastases receive their blood supply mainly from the HA, whereas liver parenchyma is primarily supplied by the portal vein. ${ }^{3}$ Additionally, fluorodeoxyuridine (FUDR), the drug most commonly used in HA infusion pump (HAIP) chemotherapy, is almost completely ( $94 \%$ to $99 \%$ ) extracted by the liver during the first pass, resulting in a hepatic-tosystemic concentration ratio of 400:1. ${ }^{4}$ Thus, HAIP chemotherapy achieves higher drug delivery directly to the tumor while minimizing systemic side effects. The HAIP also allows long-term ambulatory continuous infusion chemotherapy and avoids repetitive arterial access. 
Historically, placement of an HAIP has been performed via laparotomy..$^{2,5-9}$ This has limited the patient pool to some degree and resulted in decreased quality of life in a patient group with limited survival expectations. Recent advances in minimally invasive techniques have allowed the introduction of a laparoscopic approach to the placement of HAIPs. ${ }^{10-12}$ This study was undertaken to investigate the safety, efficacy, and early outcomes of laparoscopic HAIP (LHAIP) placement.

\section{PATIENTS AND METHODS}

\section{Patients}

From March 1998 to January 2003, 56 consecutive patients who had unresectable colorectal hepatic metastases and no evidence of extrahepatic disease on preoperative imaging studies were selected as candidates for LHAIP placement. All 38 patients who underwent LHAIP placement were reviewed. Preoperative unresectability of hepatic tumors was based on tumor number, bilobar distribution, proximity to major vascular and/or biliary structures precluding a margin-negative resection, or high risks for surgical intervention. Extrahepatic metastatic disease including lymph node involvement was considered a contraindication for pump placement. In addition to standard demographics, data collected included previous chemotherapy, tumor characteristics, perioperative data (including postoperative facts relative to the quality of life), type of hepatic arterial anatomy, management of variant hepatic arteries, intraoperative and postoperative confirmation of proper perfusion, complications (pump-related or others), duration of pump function, and data related to pump chemotherapy.

\section{Preoperative Evaluation}

Preoperative evaluation included a complete history and physical examination, routine laboratory tests (blood count and prothrombin time), biochemical profiles (bilirubin, creatinine, alkaline phosphatase, and hepatic transaminases), and serum carcinoembryonic antigen (CEA) levels. All patients underwent electrocardiography, chest X-ray, and computed tomography (CT) of the chest, abdomen, and pelvis. Thirty-seven of 38 patients $(97 \%)$ underwent ${ }^{18}$ fluorodeoxyglucose positron emission tomography (PET), which showed no evidence of extrahepatic disease. Celiac and superior mesenteric artery (SMA) arteriograms were obtained before surgery for all patients to evaluate individual hepatic arterial anatomy, to confirm patency of the portal vein, and to correlate with the operative assessment of the hepatic arterial blood supply.
Arterial anatomy was classified as normal or variant. Anatomy was considered normal if the common hepatic artery (CHA) arose from the celiac artery and then gave rise to the gastroduodenal artery (GDA) and bifurcated distally into right and left hepatic arteries. Variant HA anatomy consisted of any variation from the normal anatomy. This included replaced or accessory right or left HA, a left HA (LHA) arising early from the CHA, trifurcation anatomy (the CHA dividing into three branches simultaneously), or others (e.g., CHA arising from the SMA).

\section{Operative Technique}

The details of laparoscopic placement of HAIP have been described previously. ${ }^{10,11}$ Under general endotracheal anesthesia, all patients were placed in supine position. The surgeon stood to the patient's left and the assistant stood between the legs or to the patient's right. After a carbon dioxide $\left(\mathrm{CO}_{2}\right)$ pneumoperitoneum was created via a Veress needle or an open (Hasson's) technique, a laparoscope was placed via a $10-\mathrm{mm}$ periumbilical or right-upper-quadrant port; and then three or four ports were placed as necessary for access. Adhesions to the anterior abdominal wall were carefully taken down, and ligamentous attachments were divided as needed for access to the liver. In order to exclude extrahepatic malignant disease, the parietal and visceral peritoneal surfaces, the omentum, and the visible viscera were examined with a $30^{\circ}$ laparoscope. Laparoscopic ultrasound with a $7.5-\mathrm{MHz}$ flexible and Doppler-capable probe (Type 2002 Medical Ultrasound Scanner; B-K Medical Systems, Wilmington, MA) was performed to evaluate the size and location of the hepatic lesions and identify extrahepatic metastases, particularly porta hepatis, para-aorta, and/or celiac trunk lymph nodes. Thorough inspection and tumor staging by laparoscopy and ultrasound, together with frozen-section biopsy of all enlarged lymph nodes and suspicious extrahepatic sites, showed that 18 of 56 patients (32\%) had extrahepatic metastases, and they were excluded.

We routinely began with a cholecystectomy to avoid postoperative chemical cholecystitis. After division of the falciform and gastrohepatic ligaments and careful exposure and identification of the CHA and GDA, all small branches around the CHA were divided, starting from $1 \mathrm{~cm}$ proximal to the takeoff of the GDA and extending beyond the bifurcation of the right and left hepatic arteries. Complete devascularization of all collateral vessels to the duodenum and pancreas from the CHA and GDA was an essential component of the procedure to prevent drug misperfusion to the stomach, duodenum, and pancreas. The GDA was dissected and 
ligated 2-3 cm distal to its junction with the CHA. The beaded catheter was connected to the pump, and the pump was placed into a subcutaneous pocket. The catheter tip was passed into the abdomen with use of a tonsil-tipped clamp. The GDA was temporarily occluded proximally by a plastic bulldog clamp at its origin off the CHA. A transverse arteriotomy in the anterior wall of the GDA was created. After the catheter was trimmed to the appropriate length and inserted retrograde to the origin of the GDA, it was fixed with silk ties on either side of the bead (Fig. 1).

Although the variations in the hepatic arterial anatomy were common, the GDA cannulation technique was utilized in most cases. When a replaced or accessory left or right HA was present, it was ligated with standard placement of the catheter into the GDA. Intrahepatic collateral flow is rapidly reconstituted from the nonligated arterial supply. Replaced or accessory right hepatic arteries (RHAs) arising from the SMA were located by grasping the cystic duct stump and rotating the porta hepatis clockwise and were ligated and divided between clips. When there was a variant site of origin such as the CHA originating from the SMA or an inadequately sized GDA, alternative cannulation of the LHA was used. The LHA was ligated distally and the catheter was placed retrograde in the LHA with the tip lying at the junction of proper HA and LHA.

All pumps were implanted into a subcutaneous pocket in the right lower abdominal wall. To exclude misperfusion and confirm complete hepatic perfusion intraoperatively, $4 \mathrm{ml}$ of methylene blue in $6 \mathrm{ml}$ of normal saline was injected into the pump side port. If misperfusion of the stomach or duodenum was visualized, further intraoperative devascularization or postoperative angioembolization was indicated.

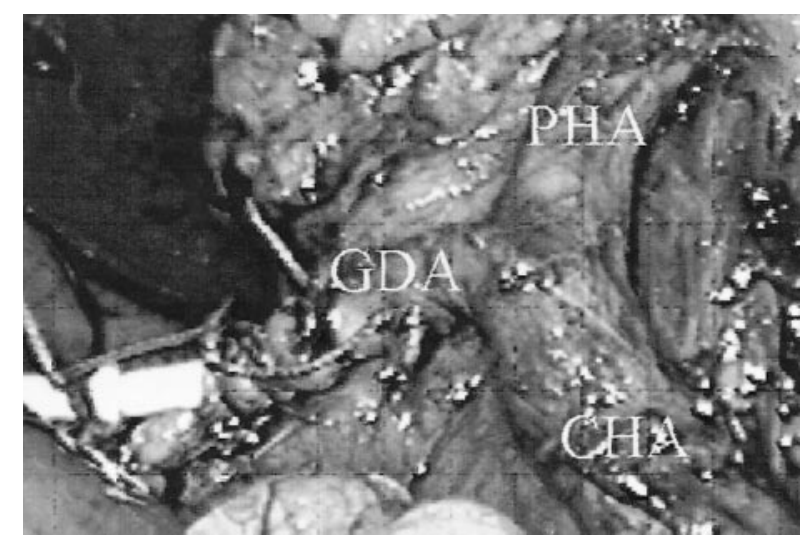

FIG. 1. Pump catheter placement and fixation (GDA, gastroduodenal artery; PHA, proper hepatic artery; $\mathrm{CHA}$, common hepatic artery).
Patients underwent LHAIP placement either as an isolated operative procedure or in conjunction with a laparoscopic radiofrequency ablation (RFA) and/or liver resection.

\section{Postoperative Care}

A technetium-99m macroaggregated albumin (TcMAA) pump flow study was performed 1 week postoperatively to verify complete hepatic perfusion and to rule out extrahepatic misperfusion prior to initiating chemotherapy. The results of these pump flow studies were recorded as normal (complete hepatic perfusion with no extrahepatic perfusion) or abnormal (incomplete hepatic perfusion or extrahepatic perfusion). A chemotherapeutic regimen of continuous administration of $0.15 \mathrm{mg} / \mathrm{kg} / \mathrm{day}$ of FUDR with $20 \mathrm{mg}$ of dexamethasone for 2 weeks, followed by infusion of heparinized saline for another 2 weeks, was recommended. Pump management was handled by the referring oncologist.

Pain medication requirement, start to ambulation, return to preoperative activity (Karnofsky performance score), and resumption of regular diet were recorded as factors of quality of life after LHAIP. Complications were classified as either pump-related or other. Pump-related complications included thrombosis (catheter or vessel), misperfusion, pocket infection, pulmonary embolus, pump replacement, and catheter migration. Pump functional times were estimated from the date of pump placement to the last follow-up, the time of death, or pump removal or documented nonfunction. Follow-up was carried out via the outpatient clinic and/or by telephone contact with oncologists or primary care doctors.

\section{Statistical Analyses}

The primary endpoints in the analysis were the occurrence of complications, length of pump functional time, and duration of survival. Survival times were calculated from both the date of pump implantation and the time of diagnosis of liver metastases to the time of death or last follow-up. Values were given as actual numbers and percentages. Continuous variables were expressed as median (range) and compared with the Mann-Whitney test; categorical variables were tested by Fisher exact test. Pump functional probabilities and overall survival rate were computed by the Kaplan-Meier method (SPSS Graduate Pack 11.5 for Windows; SPSS, Inc., Chicago, IL). Pump functional time curves were compared by $\log$-rank test. Statistical significance was accepted at $P<$ 0.05 . 


\section{RESULTS}

In this study, 18 (32\%) of 56 patients with colorectal hepatic metastases who were preoperatively selected as LHAIP candidates on the basis of conventional imaging studies were excluded by laparoscopy and laparoscopic ultrasound because of extrahepatic metastases. Ten $(56 \%)$ of 18 patients had peritoneal disease and $8(44 \%)$ had involved portal lymph nodes, para-aorta, or celiac trunk nodes. All extrahepatic metastases were confirmed by frozen-section and conventional histopathology.

A total of 38 patients underwent LHAIP; their demographic characteristics are listed in Table 1. Thirty-five of 38 patients $(92 \%)$ had previously undergone systemic chemotherapy that failed, whereas only 3 patients $(8 \%)$ had not been treated previously with chemotherapy. Twelve of the 38 patients (32\%) underwent LHAIP placement only, 24 (63\%) underwent combined LHAIP and RFA, and $2(5 \%)$ underwent concurrent laparoscopic liver resection with or without RFA. The LHAIP-only group had increased preoperative levels of alkaline phosphatase, aspartate transaminase and prothrombin time $(P \leq .02)$; higher rates of diffuse tumor pattern; and $\geq 3$ liver segments and $\geq 25 \%$ hepatic volume involvement $(P \leq .04)$ in comparison with the group having combined LHAIP with RFA and/or liver resection (Table 2).

Normal arterial anatomy was noted in 20 patients (53\%); 18 patients $(47 \%)$ had a variant type of HA anatomy. The most common site of variance was within the LHA system $(\mathrm{n}=9 ; 24 \%)$, and these variances included four replaced or accessory arteries and five LHAs originating early from the CHA (Table 3 ). One of five patients with an LHA coming off the CHA early underwent a selective LHA embolization postoperatively because of previous surgical adhesions. Six patients

TABLE 1. Patient characteristics $(n=38)$

\begin{tabular}{lc}
\hline \multicolumn{1}{c}{ Characteristic } & $\mathrm{N}(\%)$ \\
\hline Sex, male/female & $24(63 \%) / 14(37 \%)$ \\
Age, median (range), yr & $62(46-82)$ \\
Primary tumor & \\
TNM stage & $2(5 \%) / 29(76 \%) / 5(13 \%) / 2(5 \%)$ \\
T2/T3/T4/unknown & $5(13 \%) / 28(74 \%) / 5(13 \%)$ \\
N0/N1-2/unknown & $13(34 \%) / 25(66 \%)$ \\
M0/M1 & $13(8 \%) / 10(26 \%) / 25(66 \%)$ \\
AJCC stage & $24(63 \%) / 14(37 \%)$ \\
II/III/IV Location & $3(8 \%) / 32(84 \%) / 3(8 \%)$ \\
Colon/Rectum & $6(16 \%)$ \\
Tumor differentiation & $35(92 \%)$ \\
Well/Moderate/Poor & $90(70-90)$ \\
Previous liver surgery & \\
Previous systemic chemotherapy & \\
Karnofsky score, median (range) & \\
\hline
\end{tabular}

AJCC, American Joint Committee on Cancer.
TABLE 2. Preoperative laboratory data and hepatic tumor distribution

\begin{tabular}{|c|c|c|c|c|}
\hline \multirow{2}{*}{ Laboratory data } & \multicolumn{2}{|c|}{$\begin{array}{l}\text { LHAIP only } \\
\quad(\mathrm{n}=12)\end{array}$} & \multicolumn{2}{|c|}{$\begin{array}{l}\text { LHAIP with RFA } \\
\text { and/or liver } \\
\text { resection } \\
(\mathrm{n}=26)\end{array}$} \\
\hline & & & & \\
\hline CEA $<5 \mathrm{ng} / \mathrm{ml}$ & 1 & $(8 \%)$ & 8 & $(31 \%)$ \\
\hline$\geq 5 \mathrm{ng} / \mathrm{ml}$ & 11 & $(92 \%)$ & 18 & $(69 \%)$ \\
\hline $\begin{array}{l}\text { Alk phos, median } \\
\text { (range) }(\mu / 1)\end{array}$ & 149 & $(67-439)$ & 87 & $(40-201)^{a}$ \\
\hline $\begin{array}{l}\text { AST, median (range) } \\
(\mu / \mathrm{l})\end{array}$ & 45.5 & $(25-95)$ & 22 & $(16-56)^{a}$ \\
\hline $\begin{array}{l}\mathrm{TB}, \underset{(\mathrm{mg} / \mathrm{dl})}{\operatorname{median}} \text { (range) } \\
\end{array}$ & 0.7 & $(0.4-2.3)$ & 0.6 & $(0.2-1.3)$ \\
\hline $\mathrm{PT}, \underset{(\mathrm{sec})}{\operatorname{median}}$ (range) & 12.45 & $5(11-15.7)$ & 11.5 & $6(9.8-12.8)^{b}$ \\
\hline INR, median (range) & 1.1 & $(0.9-1.4)$ & 1.0 & $8(0.87-1.2)$ \\
\hline \multicolumn{5}{|l|}{ Hepatic tumor data } \\
\hline Pattern focal & 4 & $(33 \%)$ & 26 & $(100 \%)^{a}$ \\
\hline diffuse & 8 & $(67 \%)$ & 0 & $(0)$ \\
\hline Lobar unilobar & 0 & $(0)$ & 6 & $(23 \%)$ \\
\hline biolobar & 12 & $(100 \%)$ & 20 & $(77 \%)$ \\
\hline \multicolumn{5}{|l|}{$\begin{array}{l}\text { No. of segments } \\
\text { involved }\end{array}$} \\
\hline $1-2$ & 2 & $(17 \%)$ & 14 & $(54 \%)^{c}$ \\
\hline$\geq 3$ & 10 & $(83 \%)$ & 12 & $(46 \%)$ \\
\hline $\begin{array}{l}\text { Vascular involvement } \\
\text { Hepatic volume } \\
\text { involvement }\end{array}$ & 12 & $(100 \%)$ & 20 & $(77 \%)$ \\
\hline$<25 \%$ & 2 & $(25 \%)$ & 23 & $(88 \%)^{a}$ \\
\hline$\geq 25 \%$ & 9 & $(75 \%)$ & 3 & $(12 \%)$ \\
\hline
\end{tabular}

LHAIP, laparoscopic hepatic artery infusion pump; RFA, laparoscopic radiofrequency ablation; CEA, carcinoembryonic antigen; Alk phos, alkaline phosphatase; AST, aspartate transaminase; TB, serum total bilirubin; PT, prothrombin time; INR, international normalized ratio.

${ }^{a} P=0.00$

${ }^{b} P=0.02$

${ }^{c} P=0.04$

LHAIP only group vs. LHAIP with RFA and/or liver resection group.

(11\%) had a replaced or accessory RHA. The variant vessels in 17 patients required ligation at the time of LHAIP; 1 patient had a replacement-RHA embolization postoperatively because of its origin off the celiac artery and direct path behind the portal vein. Sixteen of 18 patients (89\%) with variant hepatic arterial anatomy were still able to have the GDA used for access, and the other 2 patients $(11 \%)$ underwent LHA cannulation.

In all 38 patients, the median lengths of operation, hospitalization, and blood loss were 337 minutes (range, $220-706), 3$ days (1-14), and $100 \mathrm{ml}(25-1200)$, respectively. The longest operating time (706 minutes) and largest blood loss $(1200 \mathrm{ml})$ occurred in an LHAIP with laparoscopic liver resection procedure. There were no significant differences between patients with normal and variant HA anatomy in operative time, hospital stay, and blood loss $(P \geq .20)$ (Table 4). The median postoperative requirement for pain medication was 2 days (range, 
TABLE 3. Hepatic arterial anatomy and technical management

\begin{tabular}{lccr}
\hline \multicolumn{1}{c}{ HA anatomy } & No $(\%)$ & Ligation of vessel & Catheter placement \\
\hline Normal & $20(53 \%)$ & GDA & GDA \\
Variant & $18(47 \%)$ & Replaced/Accessory RHA & \\
Replaced/accessory RHA & $6(16 \%)$ & Replaced/Accessory LHA & GDA \\
Replaced/accessory LHA & $4(11 \%)$ & LHA or GDA & GDA \\
LHA early off CHA & $5(13 \%)$ & LHA & GDA or LHA \\
CHA off SMA & $1(3 \%)$ & LHA & LHA \\
Trifurcation & $2(5 \%)$ & GDA \\
\hline
\end{tabular}

HA, hepatic artery; GDA, gastroduodenal artery; RHA, right hepatic artery; LHA, left hepatic artery; CHA, common hepatic artery; SMA, superior mesenteric artery.

${ }^{a}$ One had a selective embolization of RHA postoperatively.

${ }^{b}$ One underwent a selective embolization of LHA postoperatively.

$0-13$ ); the time to ambulation was 1 day (range, 8 hours to 8 days). Times until return to preoperative performance status and regular diet were 4 days (range, 2-21) and 2 days (15 hours to 15 days), respectively (Table 5).

There were five pump-related complications (13\%) (Table 6). Three of 38 patients (8\%) developed catheter thrombosis; 2 of them occurred late during pump chemotherapy. All three patients were treated successfully with tissue plasminogen activator. Patients with variant arterial anatomy $(2$ of $18 ; 11 \%)$ did not experience a significantly higher rate of pump-related complications in comparison with patients with normal anatomy (3 of $20 ; 15 \%)(P=1.00)$ (Fig. 2). The surgery of 1 of 38 patients $(3 \%)$ for whom LHAIP placement was planned was converted to an open procedure because of extensive adhesions from a prior open left hepatectomy. Two of 38 patients $(5 \%)$ developed an ileus requiring prolonged hospital stay. One patient (3\%) died of myocardial infarction and severe pulmonary infection on postoperative day 15. The data on LHAIP use are shown in Table 7.

The median follow-up for the 38 patients was 11 months (range, 0.5-35.5 months). Kaplan-Meier curves of the pump function showed an estimated median pump functional time of 11 months in patients with normal HA anatomy and 10 months in patients with variant anatomy $(P=.91)$ (Fig. 3). The actuarial rate of overall survival was $47.4 \%$ over the follow-up period. The estimated median survival time of all patients was 17.5 months from LHAIP placement (Fig. 4) and 27 months from diagnosis of liver metastases.

\section{DISCUSSION}

The liver is the most common site for colorectal metastasis. HAI chemotherapy has been shown to be a valid treatment for patients with colorectal liver metastases. Several randomized prospective trials have demonstrated a significant tumor response rate in comparison with that following systemic therapy. ${ }^{2}$ The median time to cancer progression was longer for HAI than for systemic chemotherapy. ${ }^{2}$ Although the issue of survival benefit in HAI chemotherapy remains uncertain primarily because many trials have had a crossover design, a few randomized studies have shown a positive effect on survival. ${ }^{13-15}$ Recently, Kemeny et al. ${ }^{16}$ demonstrated that after liver resection the 2-year actuarial rates of overall survival and hepatic disease-free survival were $86 \%$ and $90 \%$ in the HAI-with-systemic-chemotherapy arm and $72 \%(P=$ $.03)$ and $60 \%(P<.001)$ in the systemic-chemotherapyalone arm. It has also been suggested that pump placement at the time of resection and/or RFA of liver metastases to facilitate adjuvant therapy may offer better

TABLE 4. Hepatic artery anatomical, procedure-related operative data

\begin{tabular}{lcccc}
\hline \multicolumn{1}{c}{ Group } & N & $\begin{array}{c}\text { Operative time } \\
\text { median (range, min) }\end{array}$ & $\begin{array}{c}\text { Hospital stay } \\
\text { median (range, day) }\end{array}$ & $\begin{array}{c}\text { Blood loss median } \\
(\text { range, ml) }\end{array}$ \\
\hline All patients & 38 & $337(220-706)$ & $3(1-14)$ & $100(25-1200)$ \\
Normal HA & 20 & $330(250-528)$ & $3(1-14)$ & $100(25-300)$ \\
Variant HA & 18 & $355(220-706)$ & $3(1-9)$ & $125(50-1200)$ \\
LHAIP only & 12 & $300(220-480)$ & $2.5(1-9)$ & $125(25-300)$ \\
LHAIP \& RFA & 24 & $368(274-528)^{a}$ & $3(1-14)$ & $100(50-300)$ \\
LHAIP \& Res \pm RFA & 2 & $518(330-706)$ & $4.5(4-5)$ & $650(100-1200)$ \\
\hline
\end{tabular}

HA, hepatic artery; LHAIP, laparoscopic hepatic artery infusion pump; RFA, laparoscopic radiofrequency ablation; Res., laparoscopic liver resection.

There were no significant differences between normal HA group and variant HA group $(P \geq 0.20)$.

${ }^{a}$ LHAIP + RFA group vs. LHAIP group, $P=0.01$. 
TABLE 5. Factors relative to the postoperative quality of life, median (day) (range)

\begin{tabular}{|c|c|c|c|c|c|}
\hline Group & $\mathrm{N}$ & $\begin{array}{l}\text { Pain } \\
\text { requirement } \\
\text { medication }\end{array}$ & $\begin{array}{l}\text { Start to } \\
\text { ambulate }\end{array}$ & $\begin{array}{l}\text { Return to } \\
\text { preoperative } \\
\text { activity }\end{array}$ & $\begin{array}{l}\text { Resumption } \\
\text { of regular diet }\end{array}$ \\
\hline All patients & $37^{a}$ & $2(0-13 d)$ & $1(8 \mathrm{hr}-8 \mathrm{~d})$ & $4(2-21 d)$ & $2(15 \mathrm{hr}-15 \mathrm{~d})$ \\
\hline Normal HA & 19 & $2(0-7 d)$ & $1(15 \mathrm{hr}-5 \mathrm{~d})$ & $4(2-8 d)$ & $2(1-7 d)$ \\
\hline Variant HA & 18 & $2(0-13 d)$ & $1(8 \mathrm{hr}-8 \mathrm{~d})$ & $4(2-21 d)$ & $3(15 \mathrm{hr}-15 \mathrm{~d})$ \\
\hline LHAIP only & 12 & $2(1-13 d)$ & $1(1-8 \mathrm{~d})$ & $4(2-21 d)$ & $2(1-15 d)$ \\
\hline LHAIP \& RFA \pm Res. & 25 & $2(0-7 d)$ & $1(8 \mathrm{hr}-5 \mathrm{~d})$ & $4(2-8 d)$ & $2(15 \mathrm{hr}-7 \mathrm{~d})$ \\
\hline
\end{tabular}

${ }^{a}$ One death case was excluded.

HA, hepatic artery; LHAIP, laparoscopic hepatic artery infusion pump; RFA, laparoscopic radiofrequency ablation; Res., laparoscopic liver resection.

There were no significant differences between normal HA group and variant HA group $(P \geq 0.12)$, and also between LHAIP only group and LHAIP + RFA \pm Res group $(P \geq 0.32)$.

hepatic tumor control and reduce the rate of hepatic recurrence. ${ }^{11,17}$

Hepatic arterial pump chemotherapy normally requires a full laparotomy for placement of catheter and pump. Even though the reported morbidity associated with the open procedure is small, it is still considered less than desirable for this patient group. A laparoscopic approach for HAIP placement may potentially reduce the morbidity associated with laparotomy. Watkins et al. ${ }^{18}$ first described the laparoscopic approach in 1970. Since then, there have been limited reports regarding this novel procedure, which include our own results. ${ }^{10-12,19}$ The success of HAI therapy is dependent on careful patient selection and meticulous surgical technique. It is critically important that the distribution of chemotherapy reaches the entire liver without misperfusion.

Misperfusion may be intrahepatic or extrahepatic and can lead to incomplete therapy or severe complications. Intrahepatic misperfusion leads to asymmetric perfusion of chemotherapy and is attributable to accessory or replaced HA circulation that may spare portions of the liver parenchyma. To avoid intrahepatic misperfusion, ligation or embolization of these "criminal" vessels should be carried out intraoperatively or postoperatively. Ligation is safe because intrahepatic collateral arteries are developed within 2 to 4 weeks to supply the devascularized liver lobes. ${ }^{20}$

Anatomical variations of the HA are reported to occur in $30 \%$ to $50 \%$ of patients, particularly in the form of an accessory or replaced HA. ${ }^{7,8}$ In our study, 18 of 38 patients (47\%) had variant anatomy, 10 of whom had accessory or replaced arteries. It is optimal to identify these variations preoperatively with arteriograms because operative approaches may be altered by the results. These HA anomalies can pose technical challenges at the time of pump placement. Various techniques have been utilized in open procedure, including ligation or embolization of the variant vessel and cannulation of the
GDA. ${ }^{7,8,21}$ In the majority of cases, variant vessels can be successfully ligated at the time of pump placement. However, if it is not possible to ligate variant vessels intraoperatively, then selective angioembolization can be undertaken postoperatively. ${ }^{20}$

In this study, 16 of 18 patients with variant HA underwent laparoscopic cannulation of the GDA, with 14 ligations of the variant vessel at the time of LHAIP, 1 selective embolization of replaced RHA postoperatively (because this replaced artery directly arose from the celiac artery, following a retro portal-vein course), and 1 embolization of an LHA arising early from the CHA because of intensive adhesion. The remaining two patients underwent cannulation of LHA, one as a result of a small-caliber GDA and the other owing to the HA arising from the SMA, resulting in an inaccessible GDA.

Extrahepatic misperfusion of chemotherapy occurs from vessels that originate distal to the point of insertion of the catheter tip and supply the stomach and duodenum. Chemotherapy infusion can result in severe gastrointestinal symptoms. Complete, particularly posteriorly,

TABLE 6. Complications

\begin{tabular}{|c|c|c|}
\hline Type of complication & No $(\%)$ & \\
\hline \multicolumn{3}{|l|}{ Pump-related } \\
\hline Thrombosis & $3(8 \%)$ & $\begin{array}{l}\text { 2/3 occurred during } \\
\text { chemotherapy; All } \\
\text { resolved by TPA }\end{array}$ \\
\hline Pulmonary embolus & $1(3 \%)$ & $\begin{array}{l}\text { Treated successfuly by } \\
\text { anticoagulation }\end{array}$ \\
\hline Pump replacement & $1(3 \%)$ & \\
\hline Misperfusion & 0 & \\
\hline Infection & 0 & \\
\hline Catheter migration & 0 & \\
\hline \multicolumn{3}{|l|}{ Others } \\
\hline Converted to open & $1(3 \%)$ & $\begin{array}{c}\text { Due to prior left } \\
\text { hepatectomy }\end{array}$ \\
\hline Ileus & $2(5 \%)$ & $\begin{array}{l}\text { Requiring prolonged } \\
\text { hospital stay }\end{array}$ \\
\hline
\end{tabular}

TPA, tissue plasminogen activator. 


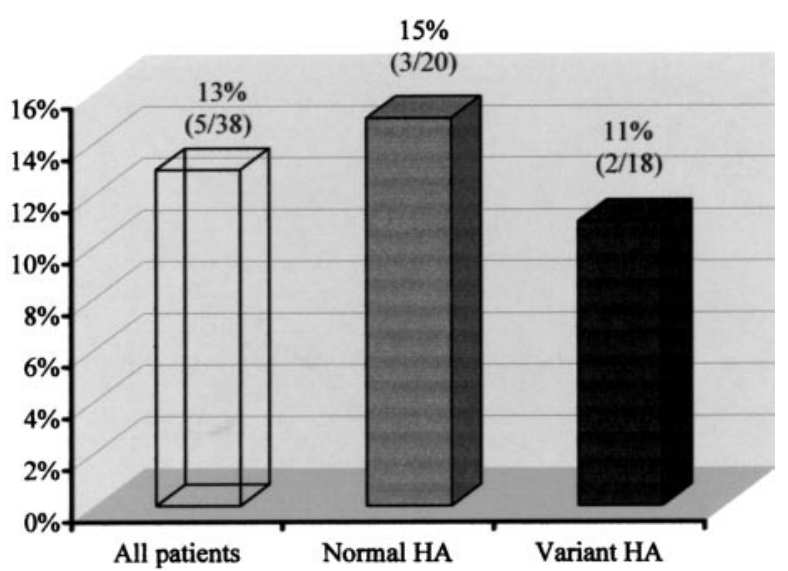

FIG. 2. Pump-related complications. There was no difference between normal and variant hepatic arterial anatomy $(P=1.00)$.

ligation and division of all branches from the HA and GDA is a critical part of the LHAIP procedure in order to prevent extrahepatic misperfusion.

To rule out misperfusion intraoperatively, we injected methylene blue into the pump side port and subsequently examined the liver, stomach, and duodenum. Before initiation of HAI chemotherapy, a Tc-MAA pump flow study can also be used to ensure absence of misperfusion and the adequacy of whole liver perfusion. Bloom et al. ${ }^{20}$ reported that the overall incidence of pump misperfusion in their series was $7.5 \%$. In our study, three patients were found to have misperfusion intraoperatively by the injection of methylene blue. Two of them had blue stain in the proximal duodenal area, and the misperfusion was corrected by laparoscopy at the time of the procedure; another patient underwent a postoperative selective embolization of a replaced RHA, as mentioned above. No misperfusion was noted in any patients by means of the

TABLE 7. Pump chemotherapy data $(n=38)$

\begin{tabular}{lc}
\hline \multicolumn{1}{c}{ Factor } & $\mathrm{N}(\%)$ \\
\hline Pump-used & $34(89 \%)$ \\
Median cycles (range) & $4(1-14)$ \\
With systemic chemotherapy & $5(15 \%)$ \\
$\quad$ Yes & $29(85 \%)$ \\
No & \\
Reasons for pump chemotherapy discontinuation & \\
$\quad$ (in 34 pump-used patients) & $3(9 \%)$ \\
Hepatic progression & $11(32 \%)$ \\
Extrahepatic progression & $4(12 \%)$ \\
Intra- and extra-hepatic progression & $3(9 \%)$ \\
Hepatic toxicity & $1(3 \%)$ \\
Reasons for pump removed & $6(16 \%)$ \\
Intrahepatic progression & $2(5 \%)$ \\
Extrahepatic progression & $1(3 \%)$ \\
Hepatic toxicity & \\
Pump chemotherapy completed & \\
\hline
\end{tabular}

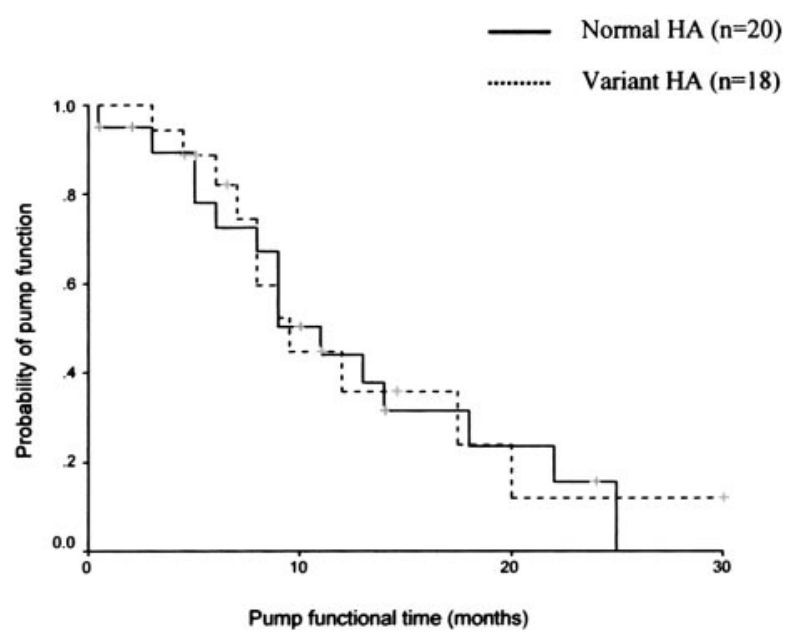

FIG. 3. Kaplan-Meier estimates of probabilities of pump function in the normal and variant hepatic arterial groups. The estimated median pump functional time was 11 months in the normal HA group and 10 months in the variant HA group $(P=.91$ by log-rank test; + indicates pump function at last follow-up).

postoperative Tc-MAA pump flow study. This suggests that methylene blue injection is a useful tool to assess misperfusion intraoperatively.

Thirty-eight patients had successful laparoscopic placement of the HAIP; in one case conversion to an open procedure was necessary because of scarring from an earlier open left hepatectomy. The operative times were long, but hospital stays were short. The reason for long operative times was multifactorial. This review includes the learning curve for principal surgeon and surgical fellows. The majority of procedures are reoperative and thus include adhesiolysis, cholecystectomy, RFA, and/or liver resection. The long operative time may

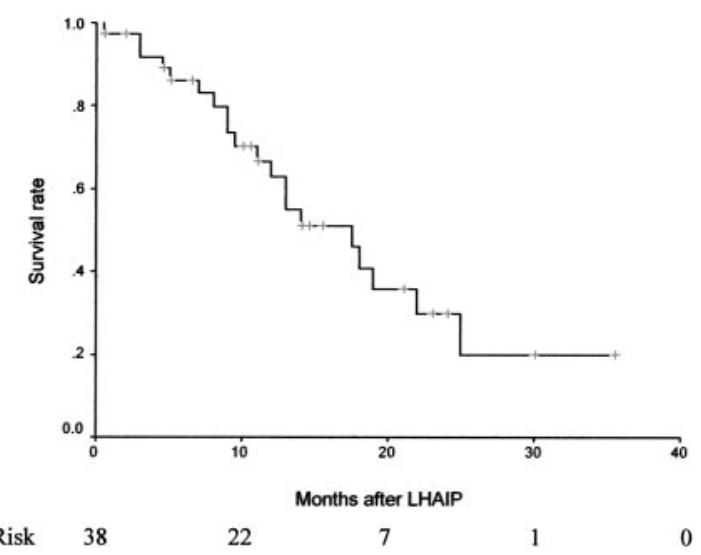

FIG. 4. Kaplan-Meier estimates of overall survival for LHAIP treatment $(n=38)$. The actuarial rate of overall survival was $47.4 \%$ and the estimated median survival time was 17.5 months, with a median follow-up of 11 months (+ indicates patients alive at last follow-up). 
have contributed to the one postoperative pulmonary embolus. Subcutaneous heparin is now added as a precautionary step in this procedure. Contrary to what might be expected, there were no differences between patients with normal and variant HA in terms of operative time, blood loss, and hospital stay ( $P \geq .20$, Table 4$)$.

Five of 38 patients (13\%) had pump-related complications in this study. There were no differences in the rates of pump-related complication $(P=1.00$; Fig. 2$)$ and pump functional times $(P=.91$; Fig. 3$)$ between patients with normal and variant HA anatomy. These findings suggest that patients with variant HA do not have increased operative time, blood loss, hospital stay, or pump complication or decreased pump functional time in laparoscopic placement of an HAIP. The same observation has been made in open procedures. ${ }^{21}$

The benefits of decreased need for postoperative pain medication and short median recovery times until ambulation, regular diet, and activity were expected in this group of patients (Table 5), although randomized controlled trials are necessary to further assess patients' quality of life between laparoscopic and open approaches with a well-validated measure of health status. As the field of minimally invasive surgery evolves, LHAIP will be more common since its technical steps remain identical to those of an open procedure and it has all the benefits of a minimally invasive approach, such as less immunosuppression, decreased pain, a shorter recovery time, and the avoidance of laparotomy in high-risk patients. ${ }^{1,10-12,19}$

Careful preoperative assessment should be performed when considering LHAIP for patients with colorectal liver metastases. Besides the routine examinations, the patient's treatment plan was determined after preoperative CT or MRI and/or PET, as well as angiography. Laparoscopic ultrasound has been an effective tool for tumor staging. Barbot et al. ${ }^{22}$ found that laparoscopic ultrasound identified unresectable disease in $25 \%$ of patients with liver tumors judged to be resectable by conventional imaging studies. Similarly, in this study, 56 patients with no extrahepatic disease revealed by preoperative imaging studies were initially taken to the operating room as candidates for LHAIP; 38 of 56 patients (68\%) eventually underwent LHAIP, and 18 patients (32\%) were excluded by laparoscopic exploration (10 for peritoneal disease; 56\%) and laparoscopic ultrasound (8 portal, para-aorta, and paraceliac lymph nodes; 44\%).

Our therapy selections for patients were based on hepatic tumor characteristics and preoperative liver function. Table 2 shows that patients who were treated with LHAIP placement only had more advanced disease and significantly worse hepatic function in comparison with patients who underwent the combined LHAIP placement with RFA and/or resection. Patients with more discrete and smaller-volume lesions but who were still not candidates for open resection were selected for combined therapy (LHAIP and RFA \pm resection). When RFA and/or resection was performed, it was always with a curative intent. No debulking procedures were intentionally performed.

The median life expectancy of patients with unresectable hepatic metastases is less than 9 months. ${ }^{9}$ Even given the advanced stage of disease and the fact that 35 of 38 patients (92\%) had previously undergone systemic chemotherapy that failed, the Kaplan-Meier survival curves (Fig. 4) showed that the actuarial rate of overall survival was $47.4 \%$ during the median follow-up of 11 months, and an estimated median time of survival was 17.5 months after LHAIP. This result is consistent with those in other similar studies..$^{9,23}$

In summary, laparoscopic placement of HAIP is a complex procedure, but with diligent attention to technical considerations, it is a feasible and safe option for treatment of hepatic metastases from colorectal cancer. Patients with a variant HA in LHAIP placement do not have increased operative time, blood loss, hospital stay, or pump complications or decreased pump functional time. Injection of methylene blue is an acceptable tool to evaluate extrahepatic misperfusion intraoperatively. Long-term studies of patients' quality of life are needed to elucidate the proper role for LHAIPs.

\section{ACKNOWLEDGMENTS}

The acknowledgments are available online in the fulltext version at www.annalssurgicaloncology.org. They are not available in the PDF version.

\section{REFERENCES}

1. Stratmann SL. Hepatic artery chemotherapy in the management of colorectal metastases. BUMC Proceedings 2002;15:376-9.

2. Kemeny N, Fata F. Hepatic-arterial chemotherapy. Lancet Oncol 2001;2:418-28.

3. Ackerman NB. The blood supply of experimental liver metastases. IV. Changes in vascularity with increasing tumor growth. Surgery 1974;75:589-96

4. Kemeny NE, Ron IG. Hepatic arterial chemotherapy in metastatic colorectal patients. Seminars Oncol 1999;26:524-35.

5. Campbell KA, Burns RC, Sitzmann JV, Lipsett PA, Grochow LB, Niederhuber JE. Regional chemotherapy devices: effect of experience and anatomy on complications. J Clin Oncol 1993;11: 822-6.

6. Kemeny N, Gones M, Sullivan D, et al. Phase I study of hepatic arterial infusion of floxuridine and dexamethasone with systemic irinotecan for unresectable hepatic metastases from colorectal cancer. J Clin Oncol 2001;10:2687-95.

7. Curley SA, Chase JL, Roh MS, Hohn DC. Technical considerations and complications associated with the placement of 180 
implantable hepatic artery infusion devices. Surgery 1993;114: 928-35.

8. Daly JM, Kemeny N, Oderman P, Botet J. Long-term hepatic arterial infusion chemotherapy: anatomic considerations, operative technique, and treatment morbidity. Arch Surg 1984;119: $936-41$.

9. Heinrich S, Petrowsky H, Schwinnen I, et al. Technical complications of continuous intra-arterial chemotherapy with 5-fluorodeoxyuridine and 5-fluorouracil for colorectal liver metastases. Surgery 2003;133:40-8.

10. Urbach DV, Herron DM, Khajanchee YS, Swanstrom LL, Hansen PD. Laparoscopic hepatic artery infusion pump placement. Arch Surg 2001;136:700-4.

11. Cheng J, Glasgow RE, O'Rourke RW, Swanstrom LL, Hansen PD. Laparoscopic radiofrequency ablation and hepatic artery infusion pump placement in the evolving treatment of colorectal hepatic metastases. Surg Endosc 2003;17:61-7.

12. Franklin ME, Gonzalez JJ. Laparoscopic placement of hepatic artery catheter for regional chemotherapy infusion: technique, benefits and complications. Surg Laparosc Endosc Percutan Tech 2002;12:398-407.

13. Rougier PR, Laplanche A, Huguier M, et al. Hepatic arterial infusion of floxuridine in patients with liver metastases from colorectal carcinoma: long-term results of a prospective randomized trial. J Clin Oncol 1992;10:1112-8.

14. Harmantas A, Rotstein LE, Langer B. Regional versus systemic chemotherapy in the treatment of colorectal carcinoma metastatic to the liver: is there a survival difference? Meta-analysis of the published literature. Cancer 1996;78:1639-45.
15. Meta-Analysis Group in Cancer. Reappraisal of hepatic arterial infusion in the treatment of nonresectable liver metastases from colon cancer. J Natl Cancer Inst 1996;88:252-7.

16. Kemeny N, Huang Y, Cohen AM, et al. Hepatic arterial infusion of chemotherapy after resection of hepatic metastases from colorectal cancer. N Engl J Med 1999;341:2039-48.

17. Vauthey JN, Marsh DW, Cendan JC, Chu NM, Copeland EM. Arterial therapy of hepatic colorectal metastases. Br J Surg 1996,83:447-55.

18. Watkins E, Khazei AM, Nahra KS. Surgical basis for arterial infusion chemotherapy of disseminated carcinoma of the liver. Surg Gyn Oncol 1970;130:581-605.

19. Feliciotti F, Paganini A, Guerrieri M, Chan R, Campagnacci R, Lzoche E. Laparoscopic intra-arterial catheter implantation for regional chemotherapy of liver metastasis. Surg Endosc 1996;10: 449-52.

20. Bloom AI, Gordon RL, Ahl KH, et al. Transcatheter embolization for the treatment of misperfusion after hepatic artery chemoinfusion pump implantation. Ann Surg Oncol 1999;6:350-8.

21. Allen PJ, Stojadinovic A, Ben-Porat L, et al. The management of variant arterial anatomy during hepatic arterial infusion pump placement. Ann Surg Oncol 2002;9:875-80.

22. Barbot DJ, Marks JH, Feld RI, Liu JB, Rosato FE. Improved staging of liver tumors using laparoscopic intraoperative ultrasound. J Surgical Oncol 1997;64:63-7.

23. Kohoe S, Endo K, Yamamoto M, et al. Protracted hepatic arterial infusion with low-dose cisplatin plus 5-fluorouracil for unresectable liver metastases from colorectal cancer. Surgery 2002;131: S128-34. 